\title{
Application of correlated basis to a description of continuum states
}

\author{
W. Horiuchi ${ }^{1, a}$ and Y. Suzuki ${ }^{2, b}$ \\ 1 Graduate School of Science and Technology, Niigata University, Niigata 950-2181, Japan \\ 2 Department of Physics, Faculty of Science and Graduate School of Science and Technology, Niigata University, \\ Niigata 950-2181, Japan
}

\begin{abstract}
In this contribution, we present a simple approach to the scattering problem using the technique for discrete states, particularly developed for a basis expansion method. An idea is very simple and the following: First, we calculate a spectroscopic amplitude (SA) between two subsystems from the "discretized" wave function obtained in the bound-state calculation. When one uses square-integrable basis, the asymptotics of the SA shows ill behavior. The behavior is easily corrected by use of the Green's function, and then phase shift is accurately determined from the SA with "correct" asymptotics. Test examples confirm the accuracy of the method. The $\alpha+n$ scattering is described using realistic nucleon-nucleon potentials. The $1 / 2^{+}$phase shifts are in reasonable agreement with experiment, and gain contributions both from the tensor and central components of the nucleon-nucleon potential. The $3 / 2^{-}$and $1 / 2^{-}$phase shifts obtained in a single-channel calculation are too small in comparison with experiment and point out the importance of including distorted configurations of the $\alpha$ particle.
\end{abstract}

\section{Introduction}

A study of many-nucleon system using a realistic interaction is very important because the interaction employed is tested strictly in comparison with experiment and thus the nuclear structure can be understood without ad hoc assumptions, and some important ingredients such as a three-body force are revealed. Of course this is not an easy task because of the complexities of the interaction and many-body problem.

Accurate solutions for discrete states of few-nucleon systems interacting via a realistic potential have been obtained with various sophisticated methods [1]. In contrast to the bound-state problem, a microscopic description of reactions using a realistic potential has been hampered by the difficulties related to continuum states as well as scattering boundary conditions. Some progress has recently been made towards the application of an $a b$ initio approach to the problem of scattering and resonances $[2,3]$. The study in this direction should be further pursued as it may provide more detailed information on the characteristics of the interaction as functions of partial waves and incident energies and on the dynamics of the participating nuclei.

Because of their apparently different natures, continuum and discrete states are obtained separately using different methods. Since, however, both of them

\footnotetext{
a e-mail: horiuchi@nt.sc.niigata-u.ac.jp

b e-mail: suzuki@nt.sc.niigata-u.ac.jp
}

are solutions of the same Schrödinger equation, it would be nice if they could be obtained on an equal footing, namely if the correlation of the constituent particles could be included in the scattering problems as well as in the bound-state problems. Several methods have in fact been proposed using square-integrable $\left(\mathcal{L}^{2}\right)$ basis functions for continuum problems in atomic and nuclear physics [4-9].

One of the methods for solving many-body problems is an expansion of the wave function in explicitly correlated bases. Recently we have extended the correlated Gaussian basis with two global vectors in order to describe more general structure [10]. The method has been applied to light nuclei and succeeded to reproduce, for example, all the observed levels of ${ }^{4} \mathrm{He}$ below $26 \mathrm{MeV}$ in a full four-body calculation using realistic nucleon-nucleon interactions [11]. It is very interesting to extend this approach to low-energy reactions and continuum problems.

In this contribution, we present a method for calculating scattering phase shifts which utilizes continuumdiscretized states obtained in a bound-state type calculation [12]. Since we use $\mathcal{L}^{2}$ basis functions for describing continuum state, the state is discretized and shows ill behavior when one goes to the asymptotic region. The wrong asymptotic behavior of the discretized state is remedied by means of the Green's function formalism.

The present approach has some similarity to that of Ref. [6], where the stochastic variational method [13$15]$ is used in conjunction with stabilization ideas to 
calculate the low energy phase-shifts for positroniumatom scattering. The basis set for describing the configuration space of the interaction region is spanned by the stochastic variational method. In Ref. [6], the phase shift is extracted by fitting the wave function in the scattering region to $\sin (k r+\delta)$. We instead use the Green's function approach to describe the wave function in the scattering region.

The basic quantity in the scattering of nuclei is the overlap integral between the product of the two internal wave functions of the nuclei and the scattering wave function of the composite system. This overlap integral is called a spectroscopic amplitude (SA) here. The phase shift is determined from the asymptotic behavior of the SA. This type of overlap integral was studied long time ago for extracting spectroscopic information from single-particle transfer reactions $[16$, $17]$.

In Sec. 2, we briefly explain our approach to fewbody problems using an explicitly correlated basis. In Sec. 3, we discuss the equation of motion which the $\mathrm{SA}$ satisfies, and we derive a formula to calculate the phase shift. The accuracy of the present approach is tested in Secs. 4.1 and 4.2. Section 4.3 presents an application to $\alpha+n$ scattering using a realistic nucleonnucleon potential model. Summary and outlook are given in Sec. 5 .

\section{Variational calculation using an explicitly correlated basis}

A variational calculation using a basis expansion method is a standard method to solve a manybody Schorödinger equation. In the basis expansion method, the wave function is expanded into finite number of non-orthogonal basis functions

$$
\Psi=\sum_{i=1}^{K} C_{i} \Phi\left(\Lambda_{i}\right),
$$

where $\Lambda_{i}$ denotes a set of variational parameters of $i$ th basis function. The $A$-nucleon system is specified by the following Hamiltonian

$$
H=\sum_{i=1}^{A} T_{i}-T_{\mathrm{cm}}+\sum_{j>i=1}^{A} v_{i j} .
$$

A three-body force is neglected in the present calculation though its inclusion does not cause any problem [10]. We believe that its inclusion would not change the basic features concerning the phase-shift of the $\alpha+n$ scattering studied here.

For the two-nucleon interaction we use AV8 type, the G3RS[18] and AV8'[19] potential models. Both of them contain central $\left(V_{\mathrm{c}}\right)$, tensor $\left(V_{\mathrm{t}}\right)$ and spin-orbit $\left(V_{\mathrm{b}}\right)$ terms. The $\boldsymbol{L}^{2}$ and $(\boldsymbol{L} \cdot \boldsymbol{S})^{2}$ terms of the G3RS potential are ignored. The ground-state properties of $d$,
${ }^{3} \mathrm{H},{ }^{3} \mathrm{He}$, and ${ }^{4} \mathrm{He}$ given by these potentials are similar to each other[10].

The quality of the variational solution depends on the choice of the basis functions. A realistic nucleonnucleon potential contains strong tensor components which induce the coupling of different orbital and spin angular momenta in the wave function of a few-nucleon system. Short range repulsive core makes convergence of a variational solution slow. The variational solution for the system faces complexities in describing the orbital motion due to these properties. One of the methods which can treat the complexities is to expand the orbital part of the wave function into an explicitly correlated bases. A variational solution for the Schrödinger equation is obtained by taking a linear combination of many basis states, which has the following form

$$
\begin{aligned}
& \Phi_{(L S) J M_{J} T M_{T}}\left(A, u_{1}, u_{2}\right) \\
& =\mathcal{A}\left\{\mathrm{e}^{-\frac{1}{2} \tilde{\boldsymbol{x}} A \boldsymbol{x}}\left[\left[\mathcal{Y}_{L_{1}}\left(\widetilde{u_{1}} \boldsymbol{x}\right) \mathcal{Y}_{L_{2}}\left(\widetilde{u_{2}} \boldsymbol{x}\right)\right]_{L} \chi_{S}\right]_{J M_{J}} \eta_{T M_{T}}\right\},
\end{aligned}
$$

where $\mathcal{Y}_{\ell}(\boldsymbol{r})=r^{\ell} Y_{\ell}(\widehat{\boldsymbol{r}})$ is a solid spherical harmonic. Here $\mathcal{A}$ is the antisymmetrizer for nucleons, $\boldsymbol{x}$ a column vector and $A$ is a $(N-1) \times(N-1)$ positivedefinite, symmetric matrix. For $\boldsymbol{x}_{i}$, we can choose any relative coordinate set, for example, Jacobi coordinate. The inner product $\widetilde{u_{1}} \boldsymbol{x}$, which we call a global vector. The global vector representation for the rotational motion used in Eq. (3) is found to be very useful. Nice property is that the basis form is unchanged under any coordinate transformations. A reader is referred to Refs. [10,20,21] for more details. The spin function $\chi_{S M_{S}}$ in Eq. (3) is specified in a successive coupling. All possible intermediate spins are taken into account in the calculation. The isospin function $\eta_{T M_{T}}$ is also treated in exactly the same way as the spin function. The variational parameters $A, u_{1}$, and $u_{2}$ are determined by the stochastic variational method[14, 15]. The number of basis $K$ is increased until the converged energy is attained.

\section{Green's function method for calculating phase-shift}

Here we give the formulation of calculating phase-shift from $\mathcal{L}^{2}$ basis functions. Let $c$ stand for a channel including a pair of nuclei denoted $\alpha_{1}$ and $\alpha_{2}$ whose normalized wave functions, $\psi_{I_{1}}\left(\alpha_{1}\right)$ and $\psi_{I_{2}}\left(\alpha_{2}\right)$, are assumed to be given. The wave function of the total system with energy $E$ generally takes the form

$$
\Psi_{J M}=\sum_{c} \Psi_{c J M}+\sum_{\gamma} \Xi_{\gamma J M}
$$

The first term on the right side stands for configurations for the elastic channel as well as other channels, whereas the second term represents the configurations 
that are needed to take account of the effects of resonances, distorted states, etc., not included in the first term. The term $\Xi_{\gamma J M}$ is thus assumed to represent the configurations in which all the nucleons are confined in the interaction region. It is assumed that $\Psi_{J M}$ satisfies the antisymmetry requirement for the exchange of the nucleons.

A key in the present method is the SA

$$
y(r)=\left\langle\Phi_{c J M}(r) \mid \Psi_{J M}\right\rangle,
$$

where $\Phi_{c J M}(r)$ is a test function for the channel $c$

$$
\Phi_{c J M}(r)=\left[\left[\psi_{I_{1}}\left(\alpha_{1}\right) \psi_{I_{2}}\left(\alpha_{2}\right)\right]_{I} Y_{\ell}\left(\hat{\boldsymbol{r}}_{c}\right)\right]_{J M} \frac{\delta\left(r_{c}-r\right)}{r_{c} r} .
$$

The coordinate $\boldsymbol{r}_{c}$ denotes the relative distance vector of the two nuclei. The angular momenta $I_{1}$ and $I_{2}$ of the two nuclei are coupled to the channel spin $I$, which is then coupled with the orbital angular momentum $\ell$ of the relative motion to the total angular momentum $J M$. In the test function (6), the two nuclei are pinned down at the relative distance specified by $r$.

The phase shift for the scattering of nuclei $\alpha_{1}$ and $\alpha_{2}$ is calculated from the asymptotic behavior of $y(r)$. When $\Psi_{J M}$ is obtained in a bound-state approximation, the SA calculated from it has ill behavior at large distances, and hence the asymptotics of $y(r)$ is usually not good enough to enable one to calculate the phase shift accurately. To resolve this problem, we derive an equation of motion which $y(r)$ calculated using the exact $\Psi_{J M}$ should satisfy. For this purpose we start from the equation

$$
\left\langle\Phi_{c J M}(r)|H| \Psi_{J M}\right\rangle=E\left\langle\Phi_{c J M}(r) \mid \Psi_{J M}\right\rangle .
$$

The Hamiltonian of the system can be decomposed into

$$
H=H_{\alpha_{1}}+H_{\alpha_{2}}+T_{c}+V_{c},
$$

where $H_{\alpha_{1}}$ and $H_{\alpha_{2}}$ are, respectively, the internal Hamiltonians of nuclei $\alpha_{1}$ and $\alpha_{2}$,

$$
T_{c}=-\frac{\hbar^{2}}{2 \mu_{c}} \frac{\partial^{2}}{\partial \boldsymbol{r}_{c}^{2}}
$$

is the kinetic energy of the relative motion between them, and

$$
V_{c}=\sum_{i \in \alpha_{1}, j \in \alpha_{2}} v_{i j}
$$

is the interaction acting between the two nuclei. If the Hamiltonian contains a three-body force, the potential $V_{c}$ acting between the nuclei should include the following terms of the three-body potentials,

$$
\sum_{\left(i \in \alpha_{1}\right)<\left(j<k \in \alpha_{2}\right)} v_{i j k}+\sum_{\left(i<j \in \alpha_{1}\right)<\left(k \in \alpha_{2}\right)} v_{i j k} .
$$

Substituting Eq. (8) into Eq. (7) and introducing a local potential $U_{c}(r)$ which acts between nuclei $\alpha_{1}$ and $\alpha_{2}$ makes it possible to transform Eq. (7) to the following inhomogeneous differential equation for $y(r)$ (the subscript $c$ in $U_{c}$ and $\mu_{c}$ is suppressed hereafter)

$$
\begin{aligned}
& {\left[\frac{d^{2}}{d r^{2}}+\frac{2}{r} \frac{d}{d r}-\frac{\ell(\ell+1)}{r^{2}}-\frac{2 \mu}{\hbar^{2}} U(r)+k^{2}\right] y(r)} \\
& =\frac{2 \mu}{\hbar^{2}}[z(r)+w(r)],
\end{aligned}
$$

where

$$
k=\sqrt{2 \mu\left(E-E_{\alpha_{1}}-E_{\alpha_{2}}\right) / \hbar^{2}},
$$

with

$$
E_{\alpha_{i}}=\left\langle\psi_{I_{i} M_{i}}\left|H_{\alpha_{i}}\right| \psi_{I_{i} M_{i}}\right\rangle,
$$

is the wave number for the relative motion, and the functions $z(r)$ and $w(r)$ are, respectively, defined by

$$
z(r)=\left\langle\Phi_{c J M}(r)\left|V_{c}-U\right| \Psi_{J M}\right\rangle,
$$

and

$$
w(r)=\left\langle\Phi_{c J M}(r)\left|H_{\alpha_{1}}-E_{\alpha_{1}}+H_{\alpha_{2}}-E_{\alpha_{2}}\right| \Psi_{J M}\right\rangle .
$$

The function $w(r)$ vanishes if $\psi_{I_{i} M_{i}}$ are the eigenfunctions of $H_{\alpha_{i}}$, that is, $H_{\alpha_{i}} \psi_{I_{i} M_{i}}=E_{\alpha_{i}} \psi_{I_{i} M_{i}}$.

Equation (12) is apparently equivalent to Eq. (7), which is the Schrödinger equation projected to the space spanned by the test function $\Phi_{c J M}(r)$. The equivalence does hold for an arbitrary choice of $U(r)$. Let $v(r)$ and $h(r)$ denote, respectively, the regular and irregular solutions of the homogeneous equation with $z(r)+w(r)$ being set to zero in Eq. (12). They are defined to satisfy the Wronskian relation, $W(v, h)(r) \equiv$ $v(r) h^{\prime}(r)-v^{\prime}(r) h(r)=1 /\left(k r^{2}\right)$. A general solution of Eq. (12) that is regular at $r=0$ and has the asymptotic behavior appropriate to the scattering solution reads

$$
y(r)=\lambda v(r)+\frac{2 \mu}{\hbar^{2}} \int_{0}^{\infty} G\left(r, r^{\prime}\right)\left[z\left(r^{\prime}\right)+w\left(r^{\prime}\right)\right] r^{\prime 2} d r^{\prime},
$$

where $\lambda$ is a constant. Here the Green's function $G$ which is a solution of the following equation

$$
\begin{aligned}
& {\left[\frac{d^{2}}{d r^{2}}+\frac{2}{r} \frac{d}{d r}-\frac{\ell(\ell+1)}{r^{2}}-\frac{2 \mu}{\hbar^{2}} U(r)+k^{2}\right] G\left(r, r^{\prime}\right)} \\
& =\frac{1}{r r^{\prime}} \delta\left(r-r^{\prime}\right),
\end{aligned}
$$

is given by [22]

$$
G\left(r, r^{\prime}\right)= \begin{cases}k v(r) h\left(r^{\prime}\right) & r \leq r^{\prime} \\ k h(r) v\left(r^{\prime}\right) & r \geq r^{\prime}\end{cases}
$$

By expressing the integral in Eq. (17) as

$$
\begin{aligned}
& \int_{0}^{\infty} G\left(r, r^{\prime}\right)\left[z\left(r^{\prime}\right)+w\left(r^{\prime}\right)\right] r^{\prime 2} d r^{\prime} \\
& =k[p(r) h(r)+q(r) v(r)]
\end{aligned}
$$


with

$$
\begin{aligned}
& p(r)=\int_{0}^{r} v\left(r^{\prime}\right)\left[z\left(r^{\prime}\right)+w\left(r^{\prime}\right)\right] r^{\prime 2} d r^{\prime} \\
& q(r)=\int_{r}^{\infty} h\left(r^{\prime}\right)\left[z\left(r^{\prime}\right)+w\left(r^{\prime}\right)\right] r^{\prime 2} d r^{\prime}
\end{aligned}
$$

the SA (17) takes the form

$$
y(r)=\left[\lambda+\frac{2 \mu k}{\hbar^{2}} q(r)\right] v(r)+\frac{2 \mu k}{\hbar^{2}} p(r) h(r) .
$$

Taking the asymptotics of this $y(r)$ determines the phase shift $\delta_{\ell}$ as

$$
\tan \delta_{\ell}=\tan \delta_{\ell}^{(0)}-\frac{2 \mu k}{\hbar^{2} \lambda} p(\infty)
$$

where $\delta_{\ell}^{(0)}$ is the phase shift corresponding to the potential scattering by $U(r)$. Let $y(r)$ of Eq. (24) (or Eq. (17)) be called SAGF (SA solved with the Green's function).

Equation (25) shows that we can obtain the phase shift accurately if $\lambda$ and $p(\infty)$ are known to high accuracy. The value of $p(\infty)$ consists of two terms, one involving the function $z\left(r^{\prime}\right)$ and the other involving the function $w\left(r^{\prime}\right)$. As was already mentioned, $w\left(r^{\prime}\right)$ vanishes if $\psi_{I_{i} M_{i}}$ are the eigenfunctions of the internal Hamiltonian $H_{\alpha_{i}}$. As we will see later, even though $\psi_{I_{i} M_{i}}$ are not completely identical with the eigenfunctions, the magnitude of $w\left(r^{\prime}\right)$ turns out to be much smaller than that of $z\left(r^{\prime}\right)$. The function $z\left(r^{\prime}\right)$ consists of the sum of various pieces of $V_{c}$ such as central, tensor and spin-orbit forces, and thus $\tan \delta_{\ell}$ can be decomposed into the contributions of those terms.

In a practical calculation of a phase shift, we have only an approximate solution for $\Psi_{J M}$, and because of this $y(r)$ as well as $z(r)$ and $w(r)$ are all approximately evaluated. The value of $\lambda$ is determined by comparing the two SAs, Eqs. (5) and (24), as will be discussed later. Evaluating $p(\infty)$ to a good approximation depends on a choice of $U(r)$. We assume that $U$ is chosen in such a way that $V_{c}$ approaches $U$ for large $r$, namely $V_{c}-U$ is an operator that is non-zero only in the interaction region. If $U$ is chosen to satisfy this condition, $z(r)$ can be accurate even though $\Psi_{J M}$ does not have a correct tail in the region where $V_{c}-U$ is negligibly small. The function $w(r)$ is accurate as well in the interaction region. Then we may assume that both $p(r)$ and $q(r)$ can be evaluated fairly accurately provided $U$ is suitably chosen.

The functions $z(r)$ and $w(r)$ (and $y(r)$ of Eq. (5)) are calculated using a code for bound-state calculations in so far as the Dirac $\delta$-function in the test function (6) is approximated as

$$
\frac{\delta\left(r_{c}-r\right)}{r_{c} r} \approx \sum_{\nu} f_{\nu}(r) f_{\nu}\left(r_{c}\right)
$$

where $\left\{f_{\nu}\right\}$ is an $\mathcal{L}^{2}$ 'pseudo-complete' set with $\left\langle f_{\nu}\right.$ $\left.f_{\nu^{\prime}}\right\rangle=\delta_{\nu \nu^{\prime}}[23]$. The expansion like Eq. (26) is used also in Ref. [3] in the basis of harmonic-oscillator functions. Because a more precise evaluation of $y(r)$ is in general desirable, an analytical method is shown in Ref. [12] to calculate the SA (5) for the correlated Gaussian basis functions $[10,14,15]$ which are employed here. The accuracy of the series expansion (26) in the calculation of functions of the type of $z(r)$ was discussed in Ref. [24] by comparing it to the exact calculation for the $\alpha+t$ system where the two fragments are assumed as the lowest shell-model states with a common oscillator parameter.

An equation similar to Eq. (12) was proposed to improve the overlap integrals which appear in nucleon transfer reactions or virtual nucleon decay $[25,26]$ and $\alpha$ decay [27]. To our knowledge, no one has yet used Eq. (12) to obtain the phase shift in a microscopic calculation.

In a microscopic reaction theory such as the resonating group method (RGM) [28], $\Psi_{c J M}$ of Eq. (4) is expressed as

$$
\Psi_{c J M}=\int_{0}^{\infty} u_{\ell}(r) \mathcal{A} \Phi_{c J M}(r) r^{2} d r
$$

where $\mathcal{A}$ is the internucleus antisymmetrizer, and the phase shift for the elastic scattering is calculated from an integro-differential equation for $u_{\ell}(r)$ [29]. We stress that $y(r)$ is used in the present approach instead of $u_{\ell}(r)$. The function $y(r)$ is subject to the simple differential equation, and it is determined uniquely regardless of whether or not Pauli-forbidden state exist, which is in contrast to the case of $u_{\ell}(r)$.

The accuracy of the phase-shift calculation crucially depends on how accurately $\lambda$ is determined. We tested two ways to determine $\lambda$. The first is to fit the SAGF (24) which is a function of $\lambda$ to the SA (5) with the least squares method in the interval $\left[r_{0}, r_{1}\right]$ where the SA (5) is expected to be accurately obtained:

$$
\text { minimize over } \lambda: \sum_{i\left(r_{0} \leq r_{i} \leq r_{1}\right)}\left[y^{\mathrm{SAGF}}\left(r_{i}\right)-y^{\mathrm{SA}}\left(r_{i}\right)\right]^{2} \text {. }
$$

We found that $\lambda$ determined in this way remains virtually unchanged within moderate choices of the interval. The second is to calculate the Wronskian $W(y, h)(r)$ using both $y^{\mathrm{SA}}(r)$ and $y^{\mathrm{SAGF}}(r)$. The latter reads

$$
W\left(y^{\mathrm{SAGF}}, h\right)(r)=\left(\lambda / k r^{2}\right)+\left(2 \mu / \hbar^{2} r^{2}\right) q(r) .
$$

Equating the two Wronskians leads to the following expression for $\lambda$ :

$$
\begin{aligned}
\lambda(r) & =k r^{2} W\left(y^{\mathrm{SA}}, h\right)(r)-\frac{2 \mu k}{\hbar^{2}} q(r) \\
& =k W\left(r y^{\mathrm{SA}}, r h\right)(r)-\frac{2 \mu k}{\hbar^{2}} q(r),
\end{aligned}
$$

which usually becomes $r$-dependent because $y^{\mathrm{SA}}(r)$ is only approximately equal to $y^{\operatorname{SAGF}}(r)$. The least 
squares fitting to this $\lambda(r)$ in the interval $\left[r_{0}, r_{1}\right]$,

$$
\text { minimize over } \lambda: \sum_{i\left(r_{0} \leq r_{i} \leq r_{1}\right)}\left[\lambda\left(r_{i}\right)-\lambda\right]^{2},
$$

yields again a stable $\lambda$ value, which is in good agreement with that determined by the first method. We use the first one mostly in what follows because it requires no differentiation of $y(r)$. Interestingly, unlike the $R$-matrix theory [30,31], our phase-shift calculation requires no channel radius.

As shown in Ref. [22], the construction of the Green's function is easy even for coupled-channel problems if $U$ is local. It is thus noted that the present approach can be straightforwardly extended to the scattering including coupled-channels.

\section{Examples}

\section{$4.1{ }^{3} S_{1} n+p$ scattering}

A first example to test the present approach is the ${ }^{3} S_{1} n+p$ scattering phase shift calculated with the Minnesota potential [32,33]. This is just a potential scattering of the two particles, and a numerically exact phase shift can easily be obtained. Diagonalizing the $n+p$ Hamiltonian with the Minnesota potential $v(r)$ in appropriate $\mathcal{L}^{2}$ basis functions produces, besides the deuteron ground state, continuum discretized states and corresponding energies $E$. The phase shifts are calculated using these states. The $\mathcal{L}^{2}$ basis functions used for the $S$ wave are Gaussians, $\exp \left(-\frac{1}{2} \beta r^{2}\right)$ with different falloff parameters $\beta$, where $\beta$ is real or complex with $\operatorname{Re} \beta>0$ [34]. In this potential problem, $y^{\mathrm{SA}}(r)$ for the discretized energy $E$ takes the form

$$
y^{\mathrm{SA}}(r)=\sum_{i=1}^{K} C_{i}(E) \mathrm{e}^{-\frac{1}{2} \beta_{i} r^{2}},
$$

and the function $z(r)$ reduces to $v(r) y^{\mathrm{SA}}(r)$, and $w(r)$ vanishes. The potential $U(r)$ is set to zero.

Table 1 compares the phase shifts calculated using Eq. (25) with those obtained with the Numerov method, which is virtually exact. The comparison shows that the present method produces very stable phase shifts which are rather insensitive to the choice of the method of determining $\lambda$ as well as the interval used for the minimization of the error. To generate the discretized states for different energies, we repeated the calculation by changing $K$ and the set of $\left(\beta_{1}, \beta_{2}, \ldots, \beta_{K}\right)$. Figure 1 displays the phase shifts obtained in this way. The phase shifts obtained with our method almost perfectly agree with those of the Numerov method in a wide range of incident energies.

\section{$4.2 \alpha+n$ scattering with an effective nucleon-nucleon potential}

In this and the following subsections we calculate the $S$ - and $P$-wave phase shifts of the $\alpha+n$ scattering in

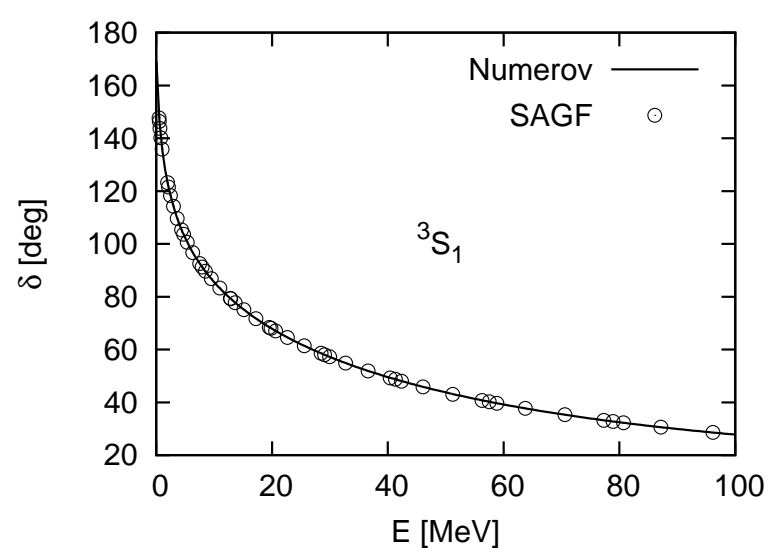

Fig. 1. Comparison of the ${ }^{3} S_{1}$ phase shifts of $n+p$ scattering between SAGF and Numerov methods. The Minnesota potential $[32,33]$ is used.

a microscopic approach. The nucleon-nucleon interaction employed in this subsection is the Minnesota potential $[32,33]$ which consists only of the central and spin-orbit potentials. The $u$ parameter of the central potential is set equal to 0.98 , and the spin-orbit potential adopted is $-591.1 \mathrm{e}^{-3 r^{2}} \boldsymbol{\ell} \cdot \boldsymbol{\sigma}$ in $\mathrm{MeV}$ (set IV of Reichstein and Tang). This potential is known to reproduce the empirical $\alpha+n$ phase shifts of Refs. [35, 36]. The phase-shift difference between the $3 / 2^{-}$and $1 / 2^{-}$states is particularly well reproduced by this spin-orbit potential, and in this sense the Minnesota potential can be regarded as an effective potential.

Only a single $\alpha+n$ channel is included in the phaseshift calculation. The wave function of the $\alpha$ particle, $\psi_{0}(\alpha)$, is obtained by diagonalizing the Hamiltonian $H_{\alpha}$ in a basis of a number of Gaussians. The binding energy with the Coulomb potential being included is $29.90 \mathrm{MeV}$ and the root-mean-square matter radius is $1.41 \mathrm{fm}$.

The relative motion function $u_{\ell}(r)$ in Eq. (27) is taken as a combination of Gaussians:

$$
u_{\ell}(r)=\sum_{i=1}^{K} C_{i} r^{\ell} \mathrm{e}^{-\frac{1}{2} \beta_{i} r^{2}} .
$$

We have calculated the phase shifts using two different methods, the microscopic $R$-matrix theory [37] and the present method. The accuracy of the $R$-matrix theory is well tested, and it is considered to produce virtually exact results to which the phase shifts of SAGF are to be compared. In the SAGF calculation the parameters $b_{i}=1 / \sqrt{\beta_{i}}$ with real $\beta_{i}$ are chosen to form a geometric progression to cover $0<b_{i} \lesssim$ $10 \mathrm{fm}$. The number $K$ of the basis functions is about 10-15. The Gaussian basis used in Eq. (34) can also be employed to construct the pseudo-complete set in Eq. (26). We obtained all the matrix elements needed in SAGF using the method developed in Ref. [10].

Figure 2 compares the SAGF phase shifts with the $R$-matrix phase shifts. The value of $\lambda$ is determined 
Table 1. Comparison of the ${ }^{3} S_{1}$ phase shifts, given in degrees, of $n+p$ scattering between Numerov and SAGF methods. The $\lambda$ value is determined using Eq. (28) (Method 1) or (32) (Method 2) with the use of different intervals $\left[r_{0}, r_{1}\right]$ (fm). The Minnesota potential $[32,33]$ is used.

\begin{tabular}{|c|c|c|c|c|c|}
\hline \multirow{2}{*}{$\begin{array}{c}E \\
{[\mathrm{MeV}]}\end{array}$} & \multirow[t]{2}{*}{ Numerov } & \multicolumn{3}{|c|}{ Method 1} & Method 2 \\
\hline & & {$[0,5]$} & {$[1,6]$} & {$[2,6]$} & {$[2,6]$} \\
\hline 0.4986 & 147.7 & 147.7 & 147.8 & 147.7 & 147.7 \\
\hline 1.959 & 123.2 & 123.3 & 123.2 & 123.2 & 123.3 \\
\hline 4.395 & 105.3 & 105.2 & 105.3 & 105.3 & 105.3 \\
\hline 7.948 & 91.2 & 91.4 & 91.2 & 91.2 & 91.1 \\
\hline 12.87 & 79.2 & 79.0 & 79.2 & 79.2 & 79.4 \\
\hline 19.54 & 68.5 & 68.7 & 68.5 & 68.5 & 68.3 \\
\hline 28.49 & 58.5 & 58.2 & 58.6 & 58.6 & 58.8 \\
\hline 40.42 & 49.3 & 49.6 & 49.3 & 49.3 & 49.1 \\
\hline 56.28 & 40.8 & 40.4 & 40.8 & 40.8 & 40.8 \\
\hline 77.31 & 33.2 & 33.6 & 33.1 & 33.2 & 33.3 \\
\hline
\end{tabular}

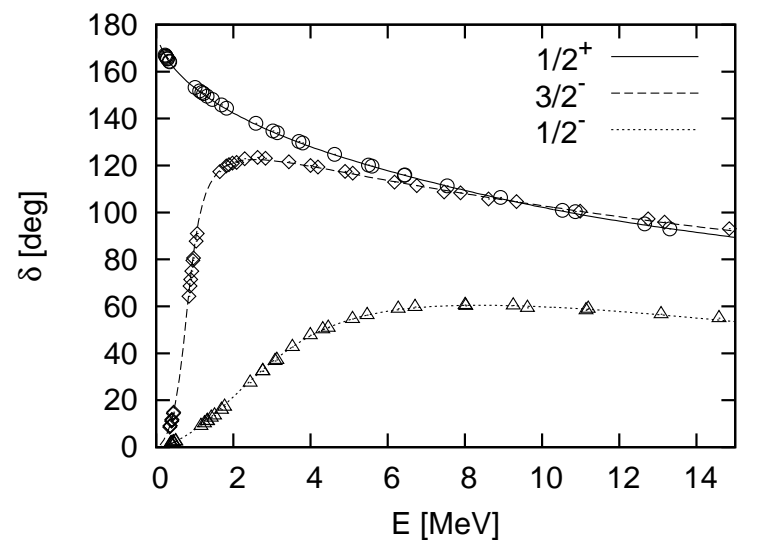

Fig. 2. Comparison of the phase shifts of $\alpha+n$ scattering between the microscopic $R$-matrix and SAGF methods. Solid, dashed and dotted lines are the phase shifts calculated with the $R$-matrix theory, while symbols are those by SAGF. The Minnesota potential $[32,33]$ is used.

by the first method using the interval $[1,5]$ (fm). The agreement is excellent. Both of $z(r)$ and $w(r)$ are included in the SAGF calculation. We switched off the function $w(r)$ and found that the phase-shift change is negligible: The largest change of a few percent occurs in the resonance region at around $1 \mathrm{MeV}$ of the $3 / 2^{-}$phase shifts. Except for this case the phase-shift change is smaller by one order of magnitude. Thus we may safely neglect the contribution of $w(r)$.

It is interesting to see how much the SAGF changes from the SA. Figure 3 compares the two SAs for the $3 / 2^{-}$state at three different energies. In each case, the SA curve agrees very well with the corresponding SAGF in the region of $r<5 \mathrm{fm}$, which indicates that the expansion (34) is good enough to describe to a good approximation the $\alpha-n$ relative motion function in the interaction region. The peak position and the amplitude of the SA curve begin to deviate from those of the SAGF curve for $r>5 \mathrm{fm}$. The deviation becomes larger as the energy increases.

\section{$4.3 \alpha+n$ scattering with realistic nucleon-nucleon potentials}

In this subsection we take the same model as in the previous subsection but employ the realistic nucleon-nucleon potentials of AV8 type, AV8' [19] and G3RS [18]. In what follows we use slightly truncated wave functions for the $\alpha$ particle to save computer time. The truncation is done by ignoring the small component with total orbital and spin angular momenta $L=1, S=1$ the magnitude of which is of the order of $0.3 \%$ or by optimizing the wave function in a smaller basis set whose size is approximately one third of the converged solution. The change of the phase shift due to the truncation is estimated to be at most a few percent.

Figure 4 displays the $S$ - and $P$-wave phase shifts obtained in an $\alpha+n$ single-channel calculation. The difference due to the nucleon-nucleon potential is not very large. The agreement between theory and experiment is quite reasonable for the $1 / 2^{+}$phase shifts. Our phase shifts agree fairly well with those of the Quantum Monte Carlo calculation obtained using only the two-body potential of AV18 [2]. In a sharp contrast to the $S$-wave phase shift, the calculated $P$-wave phase shifts are by far smaller than experiment, and considerably smaller than those obtained in Ref. [2]. We have coupled an inelastic channel of ${ }^{4} \mathrm{He}\left(0_{2}^{+}\right)+n$ with the elastic channel, but the effect of coupling is negligible below $E=15 \mathrm{MeV}$.

In Fig. 5 we show the contribution of the potential components to the $S$-wave phase shift, $\tan \delta_{0}$. Generally speaking, the two potentials give similar results. Because the spin-orbit force produces a negligible contribution, we do not show its contribution in the figure. The phase shift gets largest contributions from the central and tensor forces. For the AV8' potential, the tensor contribution is larger than the central contribution, whereas, for the G3RS potential, the central force gives a larger contribution. This is quite consistent with the relative importance of the tensor and 
$19^{\text {th }}$ International IUPAP Conference on Few-Body Problems in Physics
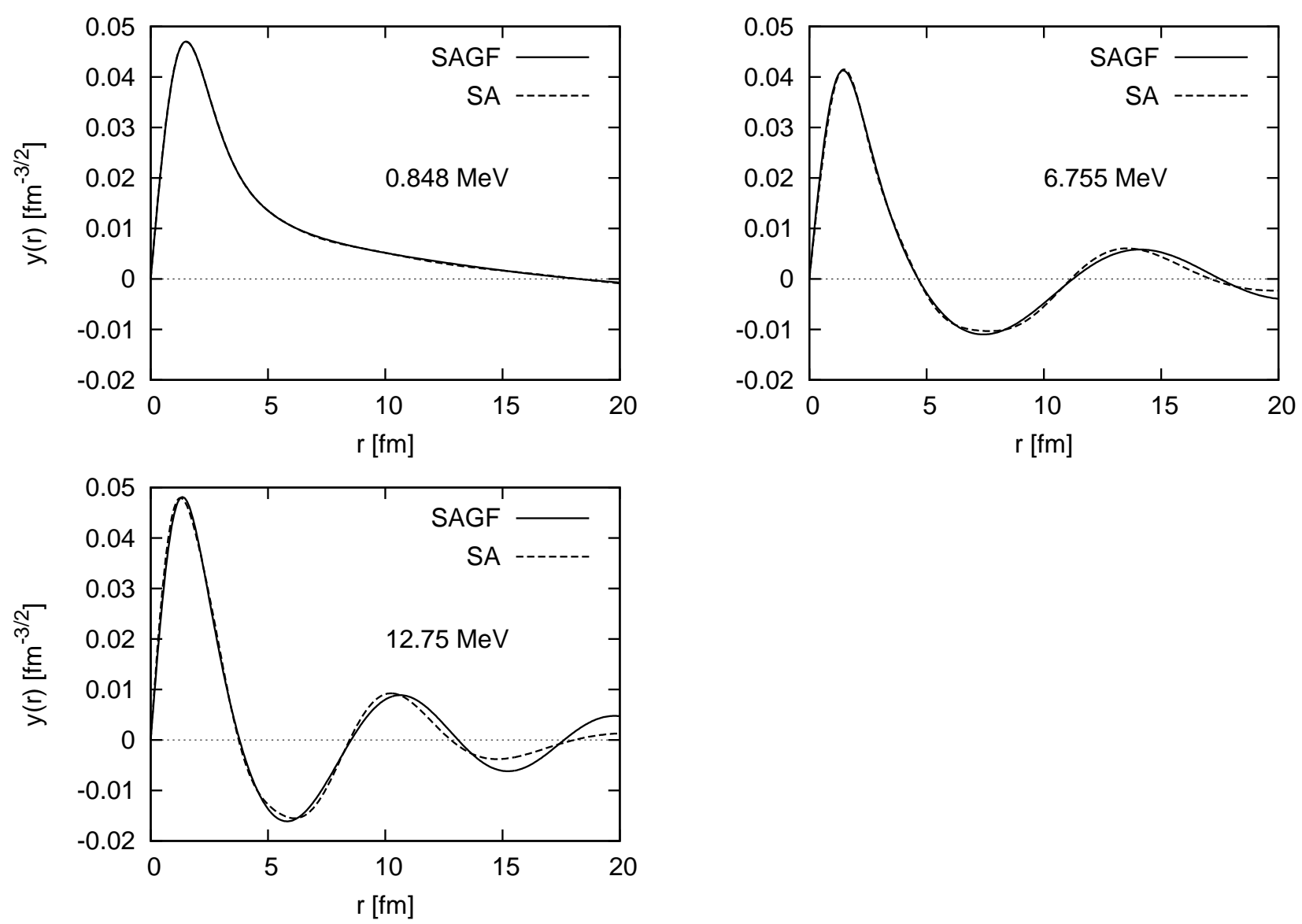

Fig. 3. Comparison between the SA and the SAGF for the $\alpha+n$ scattering $\left(3 / 2^{-}\right)$at three different energies. The Minnesota potential $[32,33]$ is used.

central forces found in the binding energies of $A=3,4$ nuclei $[10]$.

The experiment shows a $3 / 2^{-}$sharp resonance around $E=0.9 \mathrm{MeV}$ and a $1 / 2^{-}$broad resonance. The single-channel calculation apparently misses some important configurations which are relevant to the resonances, for example the inelastic channels including the excited states of ${ }^{4} \mathrm{He}$ with $J^{\pi} \neq 0^{+}$, as indicated in Ref. [3]. Other configurations which could be more important than these inelastic channels include the channels of different cluster partitions, ${ }^{3} \mathrm{H}+d$ and ${ }^{3} \mathrm{He}+2 n$, where $2 n$ denotes a di-neutron cluster. The threshold energies of these channels are lower than those of the inelastic channels. The clusters ${ }^{3} \mathrm{H}, d$, and ${ }^{3} \mathrm{He}$ may not necessarily be in their ground states but can be in their pseudo-excited states. This is plausible because the strong tensor component included in the realistic potential brings about a mixing of different orbital and spin angular momenta of the participating nuclei.

When we include the different cluster partitions of ${ }^{3} \mathrm{H}+d$ and ${ }^{3} \mathrm{He}+2 n$, we have to note the old, unresolved problem that the threshold energies of different channels are not usually reproduced well. The difference of the threshold energies between $\alpha+n$ and ${ }^{3} \mathrm{H}+d$ is $17.6 \mathrm{MeV}$ experimentally, but the theoreti- cal value turns out to be about $15 \mathrm{MeV}$ for both the AV8' and G3RS potentials. To quantify the effects of the ${ }^{3} \mathrm{H}+d$ and ${ }^{3} \mathrm{He}+2 n$ channels properly, the inconsistency in the threshold energy should be settled beforehand. The inclusion of three-body forces is important in this respect. In the calculation of Ref. [2] including the three-body force of the Illinois- 2 model, the threshold problem does not appear, and some of the distorted configurations are certainly included implicitly. However, it is not very clear whether the improvement in the phase shifts obtained with the use of the three-body force indicates simply the predominant importance of the ${ }^{3} \mathrm{H}+d$ or ${ }^{3} \mathrm{He}+2 n$ channel or the necessity of including further configurations other than the elastic channels of $\alpha+n$ and ${ }^{3} \mathrm{H}+d$. Clarifying this point will certainly be very important, but it is beyond the present work because such calculations require an expensive computation.

We here remark on similarity in the phase shifts between $\alpha+n$ and ${ }^{3} \mathrm{He}+p$ scatterings. The most attractive phase shifts of the low-energy ${ }^{3} \mathrm{He}+p$ scattering occur in the $P$-wave channel with $I=1$ and $J=2$, showing a resonance behavior. This is consistent with the fact that the ground state of ${ }^{4} \mathrm{Li}$ has $J^{\pi}=2^{-}$. The $S$-wave phase shift with $I=1$ and $J=1$ shows 

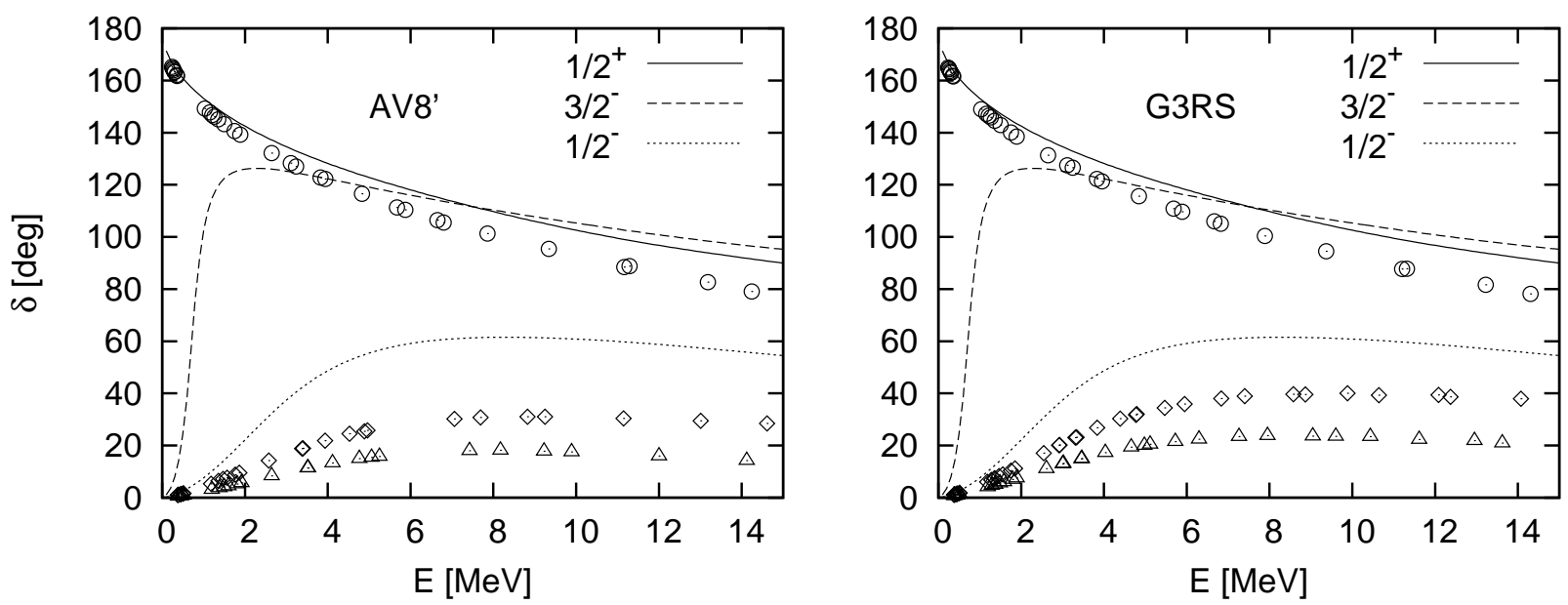

Fig. 4. $\alpha+n$ scattering phase shifts calculated using the AV8' and G3RS potentials. Solid, dashed and dotted lines are the empirical phase shifts $[35,36]$. Circle, diamond and triangle symbols denote the calculated phase shifts for $1 / 2^{+}, 3 / 2^{-}$ and $1 / 2^{-}$, respectively.
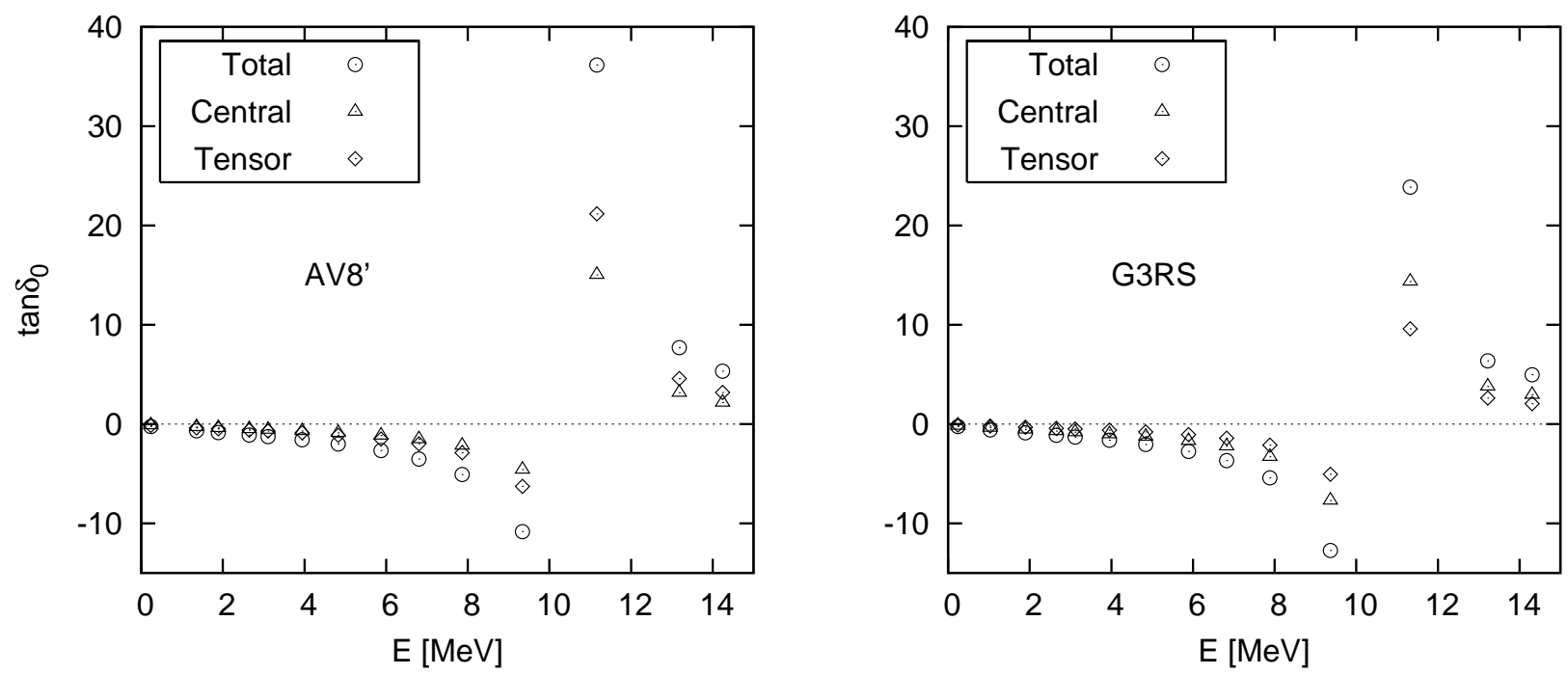

Fig. 5. Contributions of the components of the nucleon-nucleon potential to $\tan \delta_{0}$ for $S$-wave $\alpha+n$ scattering phase shifts. The contribution of the spin-orbit force is negligibly small.

a repulsive behavior similarly to the $\alpha+n$ case. The ${ }^{3} \mathrm{He}+p$ phase shifts have recently been calculated in a microscopic $R$-matrix method using the G3RS potential [38]. The result of the single-channel calculation is very similar to the $\alpha+n$ case. The $S$-wave phase shifts are accounted for fairly well in the ${ }^{3} \mathrm{He}+p$ singlechannel calculation, but the calculated $P$-wave phase shifts turn out to be too small compared to the empirical ones. A calculation of including the channels of $d\left(1^{+}, 0^{+}\right)+2 p\left(0^{+}\right)$has significantly improved the discrepancy in the phase shifts and a further inclusion of the inelastic channels of ${ }^{3} \mathrm{He}+p$ has reproduced the empirical phase shifts reasonably well. We understand this as follows. In the $P$-wave scatterings of both $\alpha+n$ and ${ }^{3} \mathrm{He}+p$, the nucleon can penetrate close to the nucleus and the nucleon-nucleus interaction, particularly the tensor force distorts the nuclear state. Thus the single-channel assumption becomes rather poor. On the other hand, in the $S$-wave case the nucleon cannot come close to the nucleus because the two identical nucleons occupying the $S$-orbit repel the incoming nucleon due to the Pauli principle, and thus the nucleus receives little distortion.

\section{Summary and outlook}

We have formulated a method to calculate the scattering phase-shift using continuum-discretized states. The spectroscopic amplitude calculated from the discretized state is compared to that obtained with the Green's function, from which the phase shift can be determined. The method has been tested successfully in the cases of $n+p$ and $\alpha+n$ scattering where two nu- 
cleons are assumed to interact via an effective nucleonnucleon potential.

Using a sophisticated wave function for the $\alpha$ particle, we have applied the present method to $\alpha+n$ scattering described by realistic nucleon-nucleon potentials. The $1 / 2^{+}$phase shifts for the $S$-wave showing a repulsive behavior of the underlying $\alpha n$ interaction are in reasonable agreement with the empirical phase shifts. The missing attraction needed to reproduce the data more perfectly is attributed to the effect of three-body forces [2]. We have analyzed how much the $S$-wave phase shifts are affected by the terms of the nucleon-nucleon potential. Both the tensor and central forces contribute significantly but the spin-orbit force has a negligible contribution.

The $P$-wave phase shifts for the $3 / 2^{-}$and $1 / 2^{-}$ states are too small with respect to experiment. This unexpected result indicates that a good reproduction of the $\alpha+n$ phase shifts attained using an effective interaction such as the Minnesota potential is an $a d$ hoc description and may not be very realistic. Based on the comparison with the result of Ref. [2], the discrepancy in the $P$-wave phase shifts could be reduced by taking into account some distorted configurations which are especially important to form the $3 / 2^{-}$resonance as well as the three-body force. A careful study on the effects of the distorted configurations will be important to pin down the contribution of the threebody force.

Finally, we would like to mention possible applications of our Green's function approach. As mentioned in the text, the method has the advantage that it can be used with ease to scattering problems of coupled-channels. Another interesting application includes a calculation of the dipole response function. Very recently we have extended our method for treating three-body continuum in the hypersperical harmonics formalism and compared in some simple cases with widely used methods using $\mathcal{L}^{2}$ basis functions, for example, the Lorentz integral transform method and the complex scaling method [39]. A study of more realistic cases and four-body continuum is very interesting and will be reported elsewhere.

\section{Acknowledgments}

W. H. is supported by a Grant-in Aid for Scientific Research for Young Scientists (No. 19.3978) as a JSPS Research Fellow for Young Scientists.

\section{References}

1. H. Kamada et al., Phys. Rev. C 64 (2001) 044001

2. K.M. Nollet, S.C. Pieper, R.B. Wiringa, J. Carlson, and G.M. Hale, Phys. Rev. Lett. 99 (2007) 022502.

3. S. Quaglioni and P. Navrátil, Phys. Rev. Lett. 101 (2008) 092501.
4. F.E. Harris, Phys. Rev. Lett. 19 (1967) 173; H.H. Michels and F.E. Harris, Phys. Rev. Lett. 19 (1967) 885 .

5. A.U. Hazi and H.S. Taylor, Phys. Rev. A 1 (1970) 1109.

6. I.A. Ivanov, J. Mitroy, and K. Varga, Phys. Rev. Lett. 87 (2001) 063201.

7. I.M. Savukov, Phys. Rev. Lett. 96 (2006) 073202.

8. J. Mitroy and M.W.J. Bromley, Phys. Rev. Lett. 98 (2007) 173001.

9. A.T. Kruppa, R. Suzuki, and K. Katō, Phys. Rev. C 75 (2007) 044602.

10. Y. Suzuki, W. Horiuchi, M. Orabi, and K. Arai, Few-Body Syst. 42 (2008) 33.

11. W. Horiuchi and Y. Suzuki, Phys. Rev. C 78 (2008) 034305.

12. Y. Suzuki, W. Horiuchi, and K. Arai, Nucl. Phys. A 823 (2009) 1-15.

13. V.I. Kukulin and V.M. Krasnopolsky, J. Phys. G 3 (1977) 795.

14. K. Varga and Y. Suzuki, Phys. Rev. C 52 (1995) 2885.

15. Y. Suzuki and K. Varga, Stochastic Variational Approach to Quantum-Mechanical FewBody Problems, Lecture Notes in Physics Monographs (Springer, Berlin, 1998), Vol. m54.

16. T. Berggren, Nucl. Phys. 72 (1965) 337.

17. W.T. Pinkston and G.R. Satchler, Nucl. Phys. 72 (1965) 641.

18. R. Tamagaki, Prog. Theor. Phys. 39 (1968) 91.

19. R. B. Wiringa, V. G. J. Stoks, and R. Schiavilla, Phys. Rev. C 51 (1995) 38.

20. Y. Suzuki, J. Usukura, and K. Varga, J. Phys. B 31 (1998) 31.

21. K. Varga, Y. Suzuki, and J. Usukura, Few-Body Systems 24 (1998) 81.

22. Y. Suzuki, T. Ando, and B. Imanishi, Nucl. Phys. A 295 (1978) 365.

23. R. Beck, F. Dickmann, and R.G. Lovas, Ann. Phys. 173 (1987) 1.

24. R.G. Lovas, K.F. Pál, and M.A. Nagarajan, Nucl. Phys. A 402 (1983) 141.

25. M. Kawai and K. Yazaki, Prog. Theor. Phys. 38 (1967) 850.

26. N.K. Timofeyuk, Nucl. Phys. A 632 (1998) 19, and references therein.

27. H. Horiuchi and Y. Suzuki, Prog. Theor. Phys. 49 (1973) 1974.

28. K. Widermuth and Y.C. Tang, A Unified Theory of the Nucleus (Vieweg, Braunschweig, 1977).

29. M. Hesse, J. Roland, and D. Baye, Nucl. Phys. A 709 (2002) 184.

30. A.M. Lane and R.G. Thomas, Rev. Mod. Phys. 30 (1958) 257.

31. R.F. Barret, B.A. Robson, and W. Tobocman, Rev. Mod. Phys. 55 (1983) 155.

32. D.R. Thompson, M. Lemere, and Y.C. Tang, Nucl. Phys. A 286 (1977) 53.

33. I. Reichstein and Y.C. Tang, Nucl. Phys. A 158 (1970) 529. 
34. E. Hiyama, Y. Kino, and M. Kamimura, Prog. Part. Nucl. Phys. 51 (2003) 223.

35. J.E. Bond and F.W.K. Firk, Nucl. Phys. A 287 (1977) 317.

36. Th. Stammbach and R.L. Walter, Nucl. Phys. A 180 (1972) 225.

37. D. Baye, P.-H. Heenen, and M. Libert-Heinemann, Nucl. Phys. A 291 (1977) 230.

38. K. Arai, S. Aoyama, and Y. Suzuki, arXiv:0812.4732.

39. Y. Suzuki, W. Horiuchi, D. Baye, arXiv:0912.1895. 